\title{
Can Necrosis and Ovarian Vein Thrombus Be a Serious Complication in Morbidly Adherent Placenta?
}

\author{
Janete Vettorazzi 1,2, Cristiano Caetano Salazar², André Bigolin 3, Gustavo dos Santos Raupp1,2, \\ Adelar Magnabosco Cosner ${ }^{4}$, Tiago Selbach Garcia ${ }^{2}$, Gustavo Schroeder ${ }^{5}$, \\ Heloísa Guedes Mussnich6, Ellen Machado Arlindo1,2
}

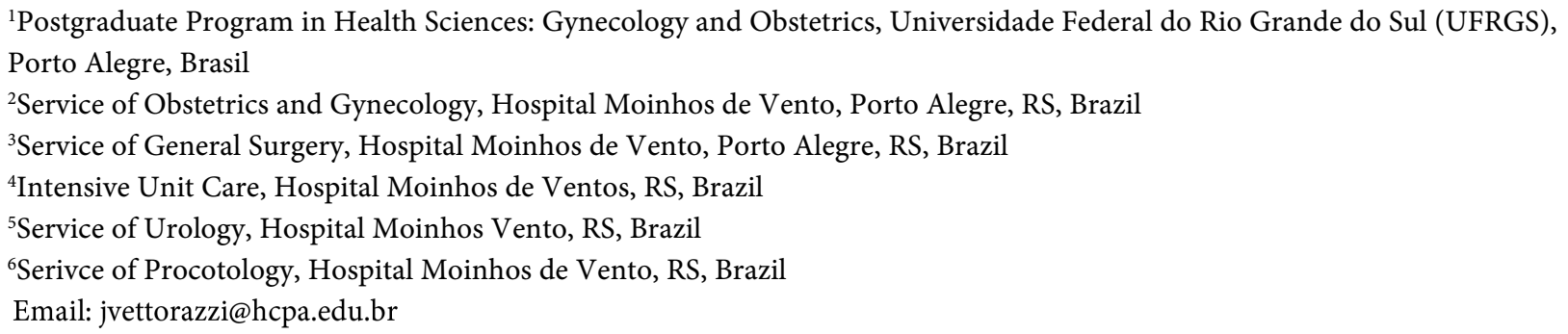

How to cite this paper: Vettorazzi, J., Salazar, C.C., Bigolin, A., dos Santos Raupp, G., Cosner, A.M., Garcia, T.S., Schroeder, G., Mussnich, H.G. and Arlindo, E.M. (2021) Can Necrosis and Ovarian Vein Thrombus Be a Serious Complication in Morbidly Adherent Placenta? Open Journal of Obstetrics and Gynecology, 11, 1477-1483. https://doi.org/10.4236/ojog.2021.1111138

Received: October 4, 2021

Accepted: November 9, 2021

Published: November 12, 2021

Copyright $\odot 2021$ by author(s) and Scientific Research Publishing Inc. This work is licensed under the Creative Commons Attribution International License (CC BY 4.0).

http://creativecommons.org/licenses/by/4.0/ (c) (i) Open Access

\begin{abstract}
We report a case of a 40-year-old woman, second pregnancy, previous cesarean section due to intrauterine growth restriction and placenta accreta spectrum disorders. She was 25 weeks gestational age, admitted to the hospital 25 weeks gestational age, diagnosed with fetal death. Initial conservative management attempt with uterine preservation progresses to complications requiring total hysterectomy followed by a rare outcome: pelvic thrombi with uterine necrosis, with its associated clinical complications.
\end{abstract}

\section{Keywords}

Morbidly Adherent Placenta, Conservative Management, Maternal Morbimortality, Placenta Accreta

\section{Introduction}

The placental insertion disorder spectrum (placenta accreta spectrum-PAS) originates at the moment of insertion of the villi which instead of adhering to the decidua, invades the myometrium, sometimes surpassing it. Failure in normal decidualization is commonly caused by uterine scarring that allows deep tissue invasion. Thus, the main risk factors are previous cesarean sections or other 
types of uterine surgery [1] [2] [3] [4] [5]. Insertion disorders can lead to serious complications, increasing maternal and fetal morbidity and mortality. In severe cases of accretism, it can affect adjacent organs such as the bladder and intestines, and an antenatal diagnosis is essential. The main consequences of abnormal placental insertion are puerperal hemorrhage, postpartum hysterectomy and complications from the surgical procedure. As for the fetus, the main complications are due to prematurity [2] [3] [4] [5].

Adequate management of PAS includes adequate prenatal diagnosis and surgical planning, in order to reduce peri- and postoperative morbidities. The most common complications are hemorrhage and need for blood products and surgical morbidities, including hysterectomy, bladder and urethral injury [1] [2] [6] [7] [8] [9] [10]. Surgical measures such as maintenance of the placenta in situ, hemostatic sutures and posterior hysterectomy and uterine artery embolization can reduce blood flow, reducing the risk of surgical morbidity.

The present report is relevant to obstetric practice as it describes the multidisciplinary management of a case of placenta accreta diagnosed just hours before the cesarean, progressing to hysterectomy and followed with a rare outcome: pelvic thrombi with uterine necrosis, with its associated clinical complications.

\section{Methods}

The patient was accompanied by an assistant physician specializing in high-risk obstetrics, a member of the team, due to the high degree of comorbidities she presented. When she was admitted to the hospital, the patient was accompanied by the team responsible for reporting the case.

\section{Case Report}

Secundigesta, 40 years old, skin color white, married, complete higher education, body mass index $22 \mathrm{~kg} / \mathrm{m}^{2}$, no prior comorbidities, with a history of previous cesarean section due to severe intrauterine growth restriction associated with a velamentous cord insertion with an intraoperative diagnosis of placental accretism in the uterine fundus. He has a history of performing several endoscopic uterine procedures for IUD removal and synechiae lysis. In the current pregnancy, she presented significant hyperemesis gravidarum, with changes in liver function tests in the first and early second trimesters. At 25 weeks of pregnancy, intrauterine fetal death was diagnosed, with no other risk factors to explain it. An investigation of placental accretism was carried out, even with ultrasonography without evidence of accretism, since the placenta was inserted in the same location as the previous accretism. Nuclear magnetic resonance (MRI) showed an area of incretism in the fundic region and to the right. Cesarean section was indicated with a view to uterine preservation, according to the patient's agreement and desire.

The cesarean was performed with difficult placental delivery, and multiple hemostatic sutures were performed, including Cho, Hartmann and B-lynch su- 
tures. Bakri's balloon was inserted before the B-lynch suture, requiring cervical dilatation to pass the balloon's exit tract, as the uterine cervix was closed. Intraoperative uterine bleeding was $1500 \mathrm{ml}$, and uterine atony responded to the use of medications (oxytocin, misoprostol, methylergometrine and tranexamic acid) and to the measures described. Hemoglobin prior to the procedure was 11.4 $\mathrm{g} / \mathrm{dL}$, decreasing afterwards to $7.7 \mathrm{~g} / \mathrm{dL}$. The patient recovered in an intensive care unit (ICU) for 24 hours, evolving well, being discharged with pharmacological prophylaxis for venous thromboembolism.

On the fourth postoperative day, she returned to the emergency room with significant abdominal pain and distension, nausea and loss of appetite, with purulent secretion in the surgical wound, requiring hospitalization in the ICU with a diagnosis of sepsis of an abdominal focus.

The clinical and gynecological evaluation did not show findings compatible with uterine infection, reaching the main diagnostic hypothesis of bacterial translocation resulting from adynamic ileum. Abdominal tomography was performed, and a small collection was identified along the isthmus of the uterine scar. Intravenous treatment with ampicillin/sulbactam and conservative measures for adynamic ileum were started. With abdominal distension and absence of bowel movements persisting, a coloproctological evaluation was performed, with no evidence of alterations on physical examination and the suspicion of an inflammatory postoperative ileus remained. A total parenteral nutrition diet was started due to oral intolerance.

Complementing the medical assessment, the general surgeon raised the suspicion of a bridle with small bowel obstruction. It was decided to perform exploratory laparoscopic surgery, whose transoperative findings showed a large amount of purulent secretion in the left iliac fossa and posterior cul-de-sac, tamponade by intestinal loops with multiple adhesions. The procedure was converted to laparotomy due to the friability of the tissues. Adhesion release, volvulus distortion, total hysterectomy with salpingectomy and ovarian preservation were performed, and thrombi were identified in pelvic vessels during the procedure. There was a bladder lesion in the urinary bladder, which was promptly corrected with raffia. The patient received two units of packed red blood cells and one unit of fresh plasma. She was kept postoperatively in the ICU, with an antibiotic change for piperacillin/tazobactan.

The anatomopathological diagnosis was extensive mural and adnexal thrombosis with uterine infarction and acute suppurative endometritis, placental remains in necrosis, acute bilateral salpingitis, acute peritonitis and parametritis and acute suppurative peri-appendicitis, and the prophylactic dose was changed to full dose of anticoagulation. The patient evolved with progressive improvement, mild liver dysfunction on parenteral nutrition and was discharged on the thirteenth postoperative day, with anticoagulation being recommended for six months. No other complications in the late postpartum period.

This case should be highlighted by the attempt to conservatively manage of placenta accreta spectrum in a patient undergoing multiple uterine surgeries 
with the aim of preserving fertility, which evolves with severe and rare complications. The patient is hospitalized for thirteenth days until discharge. In addition, the patient underwent outpatient follow-up for two years without presenting new comorbidities.

\section{Discussion}

The best outcomes in managing PAS include proper prenatal diagnosis and adequate preparation for completion of pregnancy, anticipating possible complications. The presence of a low placental attachment in the lower uterine segment associated with a history of previous uterine scarring, or even a history of multiple uterine scars or accretism, prompt ultrasound evaluation by an experienced professional, with the specific intention of looking for signs of PAS. Preoperative management includes optimization of hemoglobin values and surgical planning with a trained multidisciplinary team, availability of blood components, surgical material available for hemostasis and for conversion from cesarean to hysterectomy, in addition to predictability of recovery in ICU. The main complication of PAS is massive hemorrhage followed by coagulopathy. However, it was also seen that surgical morbidity is directly associated with the degree of invasion of the lower uterine segment, posterior bladder wall and parametrium. Less intraoperative blood loss is related to the performance of cesarean section with hysterectomy, without attempts to remove the placenta. However, conservative approaches should be considered when the degree of placental invasion is not extensive, and there is a maternal desire to preserve fertility. [6] [8] [11] [12] [13] [14]. The main conservative approaches, which are often used in combination, include partial or total non-removal of the placenta, extirpation techniques with manual placental removal, surgical resection of the area of accretism, hemostatic sutures, and the "Triple P" procedure with suture in placental area after resection [11].

Surgical complications can be life threatening, especially deep vein thrombosis. Although rare, ovarian vein thrombosis can appear as an aggravating factor for surgical procedures and cesarean sections. This complication can progress to inferior vena cava thrombosis, due to its anatomical trajectory, and pulmonary thromboembolism. The treatment, still without consensus, includes anticoagulant therapy and, sometimes, surgical reinterventions. The process can take place without any symptoms; when symptomatic, abdominal pain and fever are common. [15] [16]. In the present case, the patient had abdominal pain, but signs of necrosis and thrombosis of the pelvic vessels were only observed during the transoperative period. Thus, thrombosis of the pelvic vessels (the ovarian vein being the most commonly affected) should be included in differential diagnoses of abdominal pain in the postoperative period of women undergoing cesarean section with PAS and performing techniques for uterine preservation. In addition, although necrosis due to pelvic vessel thrombosis is a rare complication, it should be considered in cases where arterial embolization, advanced hemostasis techniques, multiple hemostatic sutures, or in cases that evolve unexpectedly, 
such as the account described here. This complication may have been partially due to multiple hemostatic sutures, associated with an eventual predisposition to thromboembolic events [17] [18] [19].

Uterine embolization has been described as an effective method, but there is still no concrete evidence for the obstetric population. Its main complications are iliac thrombosis, ischemic neuropathy, bladder gangrene, uterine necrosis, leg ischemia and buttock necrosis. In the present case, embolization was not used, but several hemostatic sutures in order to preserve the uterus according to the mother's desire. The use of multiple sutures may have contributed to thrombosis and subsequent necrosis in this case.

Despite the rare occurrence of pelvic vessel thrombosis followed by uterine necrosis, we emphasize the importance of these differential diagnoses in complications after surgical interventions, especially in the conservative management of placental disorders. In addition, we highlight the importance of multidisciplinary management with a team specialized in the care of SBP, we emphasize the importance of care in a reference center with an experienced team for the proper management.

\section{Conclusion}

The best outcomes in managing placenta accreta spectrum (PAS) include proper prenatal diagnosis and adequate preparation for completion of pregnancy, anticipating possible complications. Less intraoperative blood loss is related to the performance of cesarean section with hysterectomy, without attempts to remove the placenta. However, conservative approaches should be considered when the degree of placental invasion is not extensive, and there is a maternal desire to preserve fertility. Surgical complications can be life threatening, especially deep vein thrombosis. Although rare, ovarian vein thrombosis can appear as an aggravating factor in surgical procedures and cesarean sections. Despite the rarity of the occurrence of pelvic vessel thrombosis followed by uterine necrosis, we emphasize the importance of these differential diagnoses in complications after surgical interventions, especially in the conservative management of placental disorders.

\section{Consent}

Written informed consent was obtained from the patient for publication of this case report.

\section{Conflicts of Interest}

The authors declare no conflicts of interest regarding the publication of this paper.

\section{References}

[1] Bartels, H.C., Postle, J.D., Downey, P. and Brennan, D.J. (2018) Placenta Accreta 
Spectrum: A Review of Pathology, Molecular Biology, and Biomarkers. Disease Markers, 2018, Article ID: 1507674. https://doi.org/10.1155/2018/1507674

[2] Thurn, L., Lindqvist, P.G., Jakobsson, M., Colmorn, L.B., Klungsoyr, K., Bjarnadottir, R.I., et al. (2016) Abnormally Invasive Placenta-Prevalence, Risk Factors and Antenatal Suspicion: Results from a Large Population-Based Pregnancy Cohort Study in the Nordic Countries. BJOG, 123, 1348-1355.

https://doi.org/10.1111/1471-0528.13547

[3] Silver, R.M. and Barbour, K.D. (2015) Placenta Accreta Spectrum: Accreta, Increta, and Percreta. Obstetrics and Gynecology Clinics of North America, 42, 381-402. https://doi.org/10.1016/j.ogc.2015.01.014

[4] American College of Obstetricians and Gynecologists; Society for Maternal-Fetal Medicine (2018) Obstetric Care Consensus No. 7: Placenta Accreta Spectrum. Obstetrics \& Gynecology, 132, e259-e275.

https://doi.org/10.1097/AOG.0000000000002983

[5] Jauniaux, E., Ayres-de-Campos, D., Langhoff-Roos, J., Fox, K.A., Collins, S., Diagnosis, F.P.A., et al. (2019) FIGO Classification for the Clinical Diagnosis of Placenta Accreta Spectrum Disorders. International Journal of Gynecology \& $O b$ stetrics, 146, 20-24. https://doi.org/10.1002/ijgo.12761

[6] Pinas Carrillo, A. and Chandraharan, E. (2019) Placenta Accreta Spectrum: Risk Factors, Diagnosis and Management with Special Reference to the Triple P Procedure. Women's Health (London), 15, 1-8. https://doi.org/10.1177/1745506519878081

[7] Jauniaux, E., Collins, S. and Burton, G.J. (2018) Placenta Accreta Spectrum: Pathophysiology and Evidence-Based Anatomy for Prenatal Ultrasound Imaging. American Journal of Obstetrics \& Gynecology, 218, 75-87. https://doi.org/10.1016/j.ajog.2017.05.067

[8] Palacios-Jaraquemada, J.M., D’Antonio, F., Buca, D., Fiorillo, A. and Larraza, P. (2020) Systematic Review on Near Miss Cases of Placenta Accreta Spectrum Disorders: Correlation with Invasion Topography, Prenatal Imaging, and Surgical Outcome. The Journal of Maternal-Fetal \& Neonatal Medicine, 33, 3377-3384. https://doi.org/10.1080/14767058.2019.1570494

[9] Duzyj, C.M., Cooper, A., Mhatre, M., Han, C.S., Paidas, M.J., Illuzzi, J.L., et al. (2019) Placenta Accreta: A Spectrum of Predictable Risk, Diagnosis, and Morbidity. American Journal of Perinatology, 36, 1031-1038. https://doi.org/10.1055/s-0038-1676111

[10] Erfani, H., Fox, K.A., Clark, S.L., Rac, M., Rocky Hui, S.K., Rezaei, A., et al. (2019) Maternal Outcomes in Unexpected Placenta Accreta Spectrum Disorders: Single-Center Experience with a Multidisciplinary Team. American Journal of Obstetrics \& Gynecology, 221, 337e1-e5. https://doi.org/10.1016/j.ajog.2019.05.035

[11] Jauniaux, E., Kingdom, J.C. and Silver, R.M. (2020) A Comparison of Recent Guidelines in the Diagnosis and Management of Placenta Accreta Spectrum Disorders. Best Practice \& Research: Clinical Obstetrics \& Gynaecology, 72, 102-116. https://doi.org/10.1016/j.bpobgyn.2020.06.007

[12] Kutuk, M.S., Ak, M. and Ozgun, M.T. (2018) Leaving the Placenta in Situ versus Conservative and Radical Surgery in the Treatment of Placenta Accreta Spectrum Disorders. International Journal of Gynecology \& Obstetrics, 140, 338-344. https://doi.org/10.1002/ijgo.12308

[13] Yu, F.N.Y. and Leung, K.Y. (2020) Antenatal Diagnosis of Placenta Accreta Spectrum (PAS) Disorders. Best Practice \& Research: Clinical Obstetrics \& Gynaecology, 
72, 13-24. https://doi.org/10.1016/j.bpobgyn.2020.06.010

[14] Zuckerwise, L.C., Craig, A.M., Newton, J.M., Zhao, S., Bennett, K.A. and Crispens, M.A. (2020) Outcomes Following a Clinical Algorithm Allowing for Delayed Hysterectomy in the Management of Severe Placenta Accreta Spectrum. American Journal of Obstetrics \& Gynecology, 222, 179e1-e9.

https://doi.org/10.1016/j.ajog.2019.08.035

[15] Cao, W., Ni, X., Wang, Q., Li, J., Li, Y., Chen, T., et al. (2020) Early Diagnosis and Precision Treatment of Right Ovarian Vein and Inferior Vena Cava Thrombosis Following Caesarean Section: A Case Report. Experimental and Therapeutic Medicine, 19, 2923-2926. https://doi.org/10.3892/etm.2020.8548

[16] Yapar Eyi, E.G., Halici Ozturk, F. and Alkan, M. (2020) Placenta Percreta-Induced Uterine Rupture with Right Ovarian Vein Thrombus Protracting into the Inferior Vena Cava. Journal of Clinical Ultrasound, 48, 493-497. https://doi.org/10.1002/jcu.22903

[17] Sentilhes, L., Kayem, G., Chandraharan, E., Edwin, Palacios-Jaraquemada, J. and Jauniaux, E. (2018) FIGO Consensus Guidelines on Placenta Accreta Spectrum Disorders: Conservative Management for the FIGO Placenta Accreta Diagnosis and Management Expert Consensus Panel. International Journal of Gynecology \& Obstetrics, 140, 291-298. https://doi.org/10.1002/ijgo.12410

[18] Shamshirsaz, A.A., Fox, K.A., Erfani, H., Clark, S.L., Hui, S.K., Shamshirsaz, A.A., et al. (2019) Coagulopathy in Surgical Management of Placenta Accreta Spectrum. European Journal of Obstetrics \& Gynecology and Reproductive Biology, 237, 126-130. https://doi.org/10.1016/j.ejogrb.2019.04.026

[19] Smith, D.D., Perez-Delboy, A., Burke, W.M. and Tergas, A.I. (2016) Necrose das nádegas após embolização da artéria uterina para histerectomia tardia na placenta percreta. Case Reports in Obstetrics and Gynecology, 2016, Article ID: 6921280. https://doi.org/10.1155/2016/6921280 